\title{
Unconventional Yu-Shiba-Rusinov states in hydrogenated graphene
}

\author{
J. L. Lado ${ }^{1}$ and J. Fernández-Rossier ${ }^{1,2}$ \\ (1) International Iberian Nanotechnology Laboratory (INL), Av. Mestre José Veiga, \\ 4715-330 Braga, Portugal \\ (2) Departamento de Física Aplicada, Universidad de Alicante, 03690 Spain
}

\begin{abstract}
.
Conventional in-gap Yu-Shiba-Rusinov states require two ingredients: magnetic atoms and a superconducting host that, in the normal phase, has a finite density of states at the Fermi energy. Here we show that hydrogenated graphene can host Yu-Shiba-Rusinov states without any of those two ingredients. Atomic hydrogen chemisorbed in graphene is known to act as paramagnetic center with a weakly localized magnetic moment. Our calculations for hydrogenated graphene in proximity to a superconductor show that individual adatoms induce in-gap Yu-Shiba-Rusinov states with an exotic spectrum whereas chains of adatoms result in a gapless $\mathrm{Yu}$ Shiba-Rusinov band. Our predictions can be tested using state of the art techniques, combining recent progress of atomic manipulation of atomic hydrogen on graphene together with the well tested proximity effect in graphene.
\end{abstract}




\section{Introduction}

Magnetic moments have long been known[1, 2] to deplete the superconducting order, hindering the coexistence of ferromagnetism and superconductivity. At the atomic scale, a single magnetic atom can locally modify the superconducting order parameter, binding ingap Yu-Shiba-Rusinov (YSR) states[2, 3, 4, 5]. Using scanning tunneling spectroscopy (STS), these in-gap states have been observed in a variety of systems $[6,7,8,9,10]$, all of them involving transition metal or rare earth local moments. In presence of either spin-orbit coupling or non-collinear magnetic order, chains of YSR impurities[11] have been predicted to result in topological superconductivity, whose fingerprint would be the emergence of zero energy Majorana[12] edge states. The recent observation[13] of zero energy end states by means of STS in atomic chains of $\mathrm{Fe}$ atoms on the surface of superconducting $\mathrm{Pb}$ has been interpreted along this line and has triggered and enormous interest in the engineering of YSR states[11, $14,15]$.

In conventional superconductors, the parameter that controls the energetics of YSR states is $\rho J$ where $\rho$ is the density of states (DOS) at the Fermi surface in the normal phase and $J$ is strength of the Kondo exchange between the local moment with spin $S$ and the conduction electrons. When $\rho$ is a slowly varying function of energy, the binding energy of YSR states for a classical spin in a superconductor, is given by $[2,16]$

$E_{S}=\Delta \frac{1-\left(\pi \frac{S}{2} J \rho\right)^{2}}{1+\left(\pi \frac{S}{2} J \rho\right)^{2}}$

where $\Delta$ is the superconducting energy gap. Thus, according to this classical result, a finite $\rho$ is needed in order to have in-gap YSR states. We now address the question of how could graphene change this state of affairs.
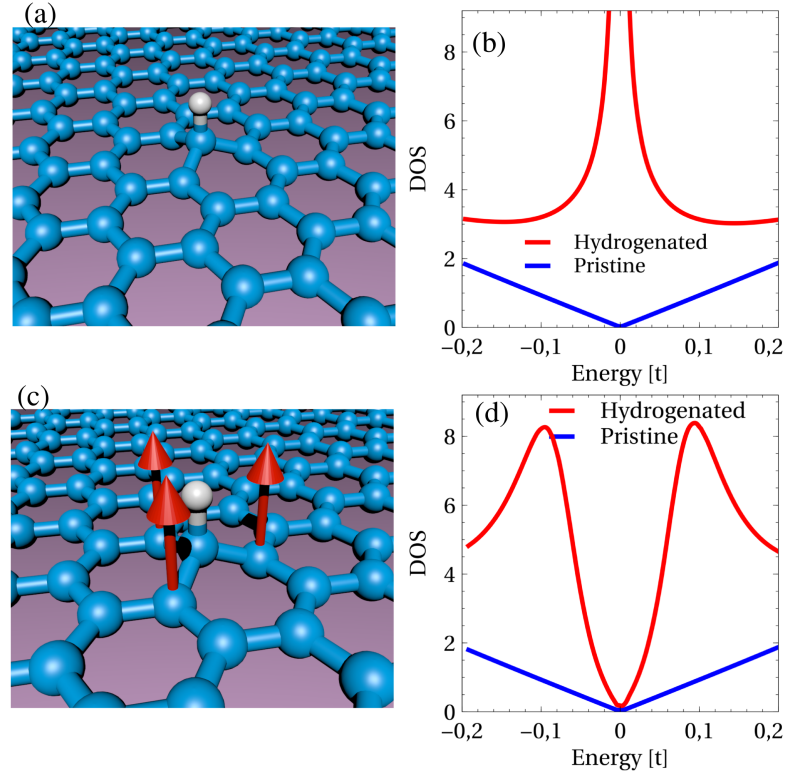

(e)
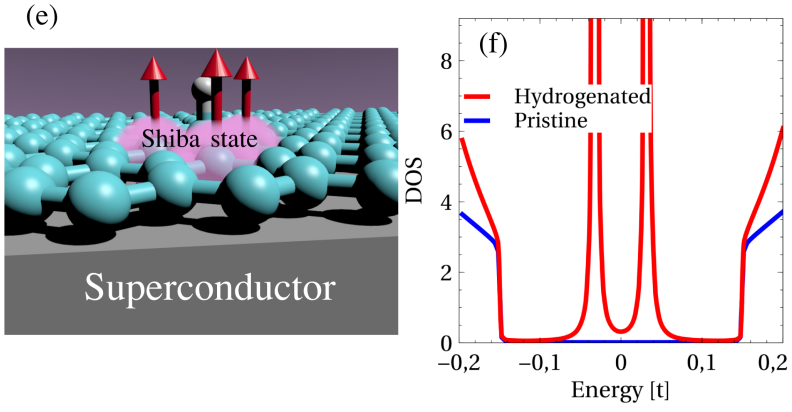

Figure 1. (a) Sketch of hydrogenated graphene (b) and DOS in the carbons close to the hydrogen. Upon introduction of interactions a local magnetic moment is developed (c), changing dramatically the associated DOS (d). When the system is placed on top of a superconductor (e), YSR excitations arise below the superconducting gap (f). The parameters used are $\Delta=0.15 t$ and $J=0.4$.

Graphene can be made superconductor via proximity effect using several complementary strategies. On one hand superconductivity can be induced in lateral graphene/superconductor heterostructures[17, 18, 19] and on the other hand taking advantage of its two dimensional character, graphene can be deposited on top of and beneath a superconductor[20, 21]. The recent reports of fabrication of vertical Van der Waals structures 
combining[22, 23, 24] graphene with superconducting $\mathrm{NbSe}_{2}$ also present a promising venue in this regard. Moreover, a carbon layer of intercalated graphite $C_{6} C a,[25,26,27]$ could be considered as superconducting graphene, although in the former case the Fermi level is away from half filling.

Local magnetic moments can be induced in graphene without using transition metals via chemisorption of atomic hydrogen $[28,29]$ as well as many other covalent functionalizations[30, 31]. Within a one-body picture, the chemisorption of atomic hydrogen in graphene creates a zero energy state, which greatly enhances the local DOS close to the Fermi energy. Electron-electron interactions result $[28,32,29,33]$ in the formation of a local moment associated to chemisorbed hydrogen. When two hydrogen atoms are chemisorbed on the same sub lattice, ferromagnetic couplings are expected[29, 33]. These theoretical results are in line with recent experiments[34] where both individual chemisorbed hydrogen, as well as dimers and trimers, have been probed using STS. In these experiments atomic manipulation of individual hydrogen atoms has been demonstrated, showing the potential for atomic scale engineering of magnetism in graphene. In addition, this magnetism can be turned on and off when the density of carriers is changed [34], in line with the experimentally demonstrated electrical control of paramagnetism in the case of fluorinated graphene [35] and as expected from theoretical calculations[36].

\section{Methods}

We now model hydrogenated graphene on top of a superconductor using a one orbital tight-binding model with pairing and exchange fields. Within the one-orbital model, the (a)
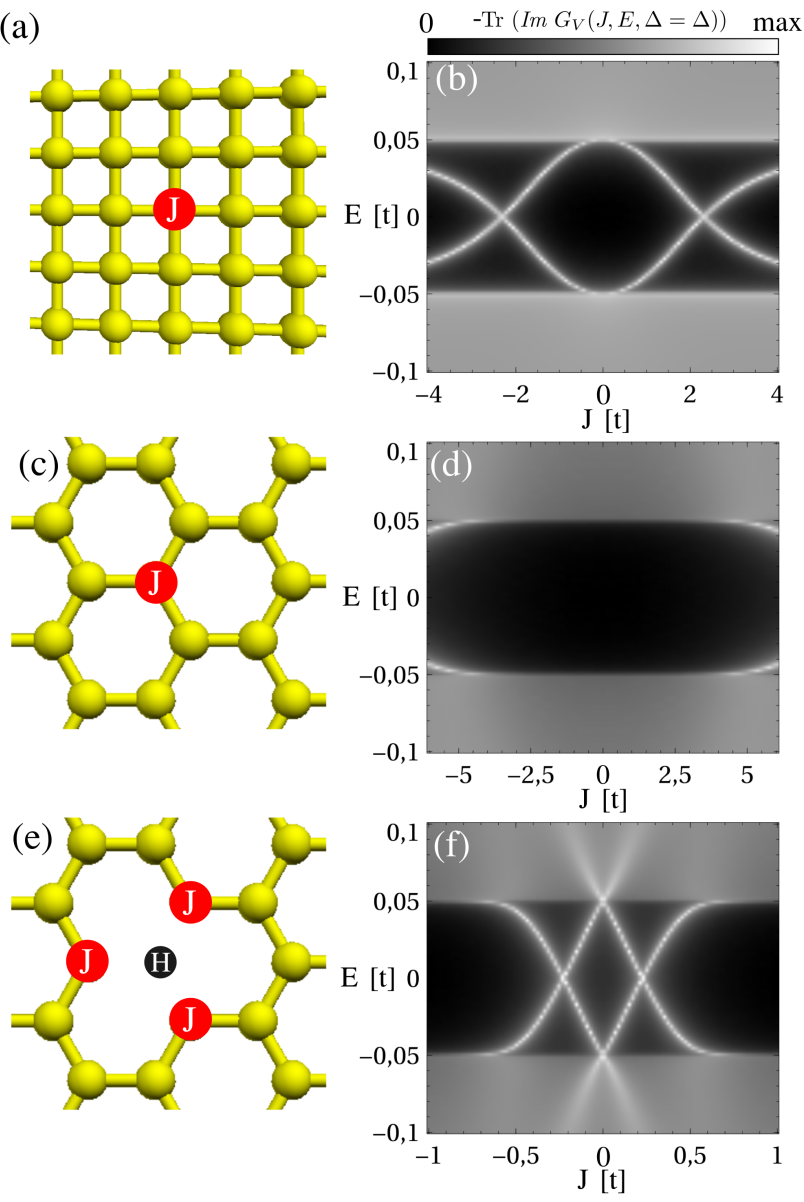

Figure 2. Single magnetic impurity in an infinite square lattice (a), showing in-gap states described by Eq.1. The same impurity in the honeycomb lattice (c) does not show YSR states at weak coupling (d). For the single hydrogenated graphene (e), a new and qualitatively different branch of in-gap state arises (f).

effect of hydrogenation and other covalent functionalizations[31] are equivalent to the removal of a site in the lattice[33] without modifying the onsite energies of neighboring carbon atoms. At the non-interacting level, this results in an in-gap $E=0$ state in the case of gapped graphene nanostructures, and a resonance in the case of $2 \mathrm{D}$ graphene [37]. In most instances, interactions have been included at a mean field level using supercells[28], that invariably result in spinsplit solutions with a sublattice polarized magnetization cloud in the neighborhood of 
the hydrogen atom and total spin $S=$ 1/2. Our Hamiltonian includes the spindependent potential in the three closest carbon atoms to the one underneath the chemisorbed hydrogen. This minimal model mimics a self-consistently calculated exchange field that breaks time reversal symmetry that implies a local magnetization induced by the chemisorbed hydrogen. Finally, in order to account for the proximity induced superconducting gap $\Delta$, we include a pairing term[38] in the theory. Thus, the complete Bogoliubov-De Gennes (BdG) Hamiltonian reads:

$H=H_{k i n}+H_{W}+H_{J}+H_{S C}$

where $H_{k i n}$ describes hopping, $H_{W}$ an onsite potential term, $H_{J}$ is the exchange term and $H_{S C}$ the superconducting pairing. The hopping term is the standard nearest neighbor (NN) hopping:

$H_{k i n}=t \sum_{s} \sum_{(i, j) \in N N} c_{j \sigma}^{\dagger} c_{i \sigma}$

In the case of the hydrogenated system, the effect of hydrogenation is captured by adding an infinite onsite energy $W$ to functionalized carbon site

$H_{W}=\lim _{W \rightarrow \infty} W \sum_{s} c_{0, s}^{\dagger} c_{0, s}$

The exchange term can be written down as:

$H_{J}=\sum_{i} j(i) c_{i \sigma}^{\dagger} \frac{\sigma_{z}}{2} c_{i \sigma}$

When we model the a conventional Shiba state in the square and honeycomb lattice, we take $W=0$ and $j(i)$ takes a non-zero value $J$ in just one site, marked in red in figures 2(a,c). In contrast, when we model hydrogenated graphene we take $W=\infty$ and $j(i)$ takes a non zero value $J$ in the three first neighbors of the functionalized carbon site (see figure 2(e)). We treat $J$ as a free parameter, to account for variations of the local magnetization coming from temperature or doping $[35,36]$.

Finally, the superconducting proximity effect is introduced as an effective conventional s-wave pairing term

$H_{S C}=\Delta \sum_{i}\left[c_{i, \uparrow} c_{i, \downarrow}+c_{i, \downarrow}^{\dagger} c_{i, \uparrow}^{\dagger}\right]$

Here $\Delta$ is taken as an input parameter in the calculation, and no attempt to compute it self-consistently is done. Since we are considering a single impurity in an infinite pristine system, we have to deal with a problem with infinite size and no translational invariance. We tackle the problem using Green functions and a partition method, valid for any dimension. To do so, we divide the problem in a core region that contains the impurity site(s) and an outer region[39]. This division is performed by creating a graphene supercell as the new unit cell $C$, where the central supercell host the hydrogenated site and the sites with exchange coupling

$h_{V}=h_{k i n}+h_{W}+h_{J}+h_{S C}$

with $h_{k i n}, h_{W}, h_{J}$ and $h_{S C}$ the projection of Eq. 2 in the central defective supercell. The rest of the system is formed of pristine supercells coupled to each other and to the defective one. To calculate the full Green function of the defective supercell, we write down the Dyson equation of defective supercell coupled to the infinite graphene.

$G_{V}(E)=\left(E-h_{V}-\Sigma(E)\right)^{-1}$

where $\Sigma(E)$ is the selfenergy induced over the defective supercell by the rest of pristine system. The calculation of $\Sigma(E)$ is done noting that, for a pristine supercell, an analogous Dyson equation can be written up:

$G_{0}(E)=\left(E-h_{0}-\Sigma(E)\right)^{-1}$ 
However, in the case of pristine graphene the Green function $G_{0}$ of the supercell $C$ can also be calculated by summing up the Green functions of the Bloch Hamiltonian $H_{k}$ as

$G_{0}(E)=\frac{1}{(2 \pi)^{2}} \int\left(E-H_{k}\right)^{-1} d^{2} k$

with $H_{k}$ the usual Bloch Hamiltonian

$H_{k}=h_{0}+\sum_{\vec{r}} t_{\vec{r}} e^{i \vec{k} \cdot \vec{r}}$

where $h_{0}$ is the pristine intracell hopping matrix, $t_{\vec{r}}$ are the intercell hopping matrices and $\vec{r}$ are real space vector connecting supercells. Coming back to Eq. 9, the self energy that pristine graphene induces on a central supercell can be calculated combining Eq. 9 and 10 as

$\Sigma(E)=E-h_{0}-\left(\frac{1}{(2 \pi)^{2}} \int\left(E-H_{k}\right)^{-1} d^{2} k\right)^{-1}$

Finally, with the previous self energy the full Green function of the defective supercell $G_{V}$ can be calculated with Eq. 8. From the Green function, the spectral function can easily be obtained as $\rho=-\frac{1}{\pi} \operatorname{Im} G_{V}(E)$ introducing a small but finite imaginary part in the energy $E \rightarrow E+i \delta$. We stress that this method is specially well suited to capture single impurities in infinite systems, not relying on periodic boundary conditions and avoiding undesired interference effects between different impurities.

\section{Results}

It is instructive to analyze the density of states of the single hydrogenated graphene in three stages. First, with $J=\Delta=0$, we see in figure 1(b) how the density of states diverges for $E=0$, in line with analytic results [40]. Second, when the effect of the mean field exchange is added ( $J \neq 0$ in our Hamiltonian), the $E=0$ peak spin splits, and results in a
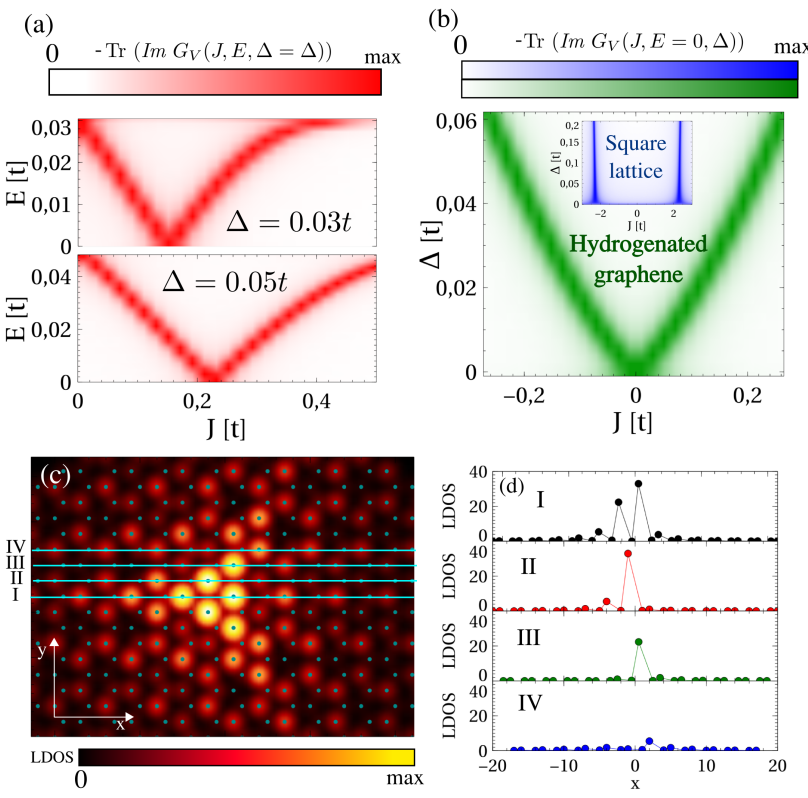

Figure 3. (a) Low energy spectral function of the YSR states for single hydrogenated graphene, for different paring couplings, showing an anomalous displacement of the pairing switching point. (b) $E=0$ spectral function as a function of superconducting paring and exchange coupling for hydrogenated graphene (green), showing a non linear dependence of the switching point. The inset (blue) shows the result for the usual parabolic band which yields a $\Delta$ independent transition point. (c) Spatially resolved DOS at $E=0$ for the parity switching point $J=0.22 t, \Delta=0.05 t$. Panel (d) shows the amplitude of the DOS along different lines, marked in panel (c), showing a strong localization of the YSR state close to the hydrogenated site. In panel (c), the hydrogen adatom is deposited in the center of the triangular pattern.

vanishing DOS at $E=0$ (see Fig. 1(d)). The resulting DOS calculated using the embedding method shows a phenomenology analogous to a toy model, a zero energy level, spin splitted by an exchange $J$ and coupled to a bath with the graphene density of states $\rho_{0}=\lambda|E|$. In this toy model, the Dyson equation gives the spectral function $\rho_{ \pm}(E, J)=\frac{\lambda|E|+0^{+}}{(J \pm E)^{2}+\left(\lambda|E|+0^{+}\right)^{2}}$. The dramatic difference between the results with $J=0$ (Fig. 1b) and $J \neq 0$ (Fig. 1d) are analogous to the pathological behavior of the function $\rho(E, J)$. In particular, $\rho$ can not 
be Taylor expanded for $E=0$, because the small $J$ and $E$ limits can not be exchanged. This prevents the use of $\rho J$ as a well defined function and invalidates the use of Eq.1 to model YSR states in hydrogenated graphene.

Finally, in the third stage, we study the effect of the superconducting proximity effect on graphene. A proximity gap opens in the DOS of pristine graphene (blue line in Fig. $1(\mathrm{f}))$. In contrast, the calculated DOS close to a chemisorbed hydrogen atom shows an intragap YSR state (red line in Fig. 1(f))

\subsection{YSR states for individual magnetic centers}

The in-gap excitation energy is governed by the strength of the exchange coupling. Using our methodology for a magnetic impurity embedded in square lattice (Fig. 2a), with conventional parabolic bands, the evolution of the in-gap YSR state as a function of $J$ is shown in Fig. 2b, with $\Delta=0.05 t$ taking chemical potential $E_{F}=-2 t$. Our results follow Eq. 1. In particular, in the low $J$ limit the in-gap energies $E_{S}$ follow a quadratic law $\Delta-E_{S} \propto J^{2}$.

In contrast, a single magnetic impurity (Fig. 2c) in the honeycomb lattice at half filling yields no in-gap state unless the exchange interaction $J$ takes unrealistically large values $J>>t$ (Fig. $2 \mathrm{~d}$ ), in line with a previous result[41]. This can be understood within the standard model as a straightforward consequence of the vanishing DOS at $E=0$. The situation is radically different when we consider the model for hydrogenated graphene (Fig. 2e). In this case, in-gap YSR states appear at weak coupling that, in contrast with conventional YSR states, follow a linear evolution with $J$ at low coupling, and therefore are not described by Eq. 1. This is the main result of this paper.

On top of their linear dependence on $J$, the hydrogenated graphene YSR states have another unconventional property. Let us define $J_{c}$ as the point that satisfies $E_{S}\left(J_{c}\right)=0$, which marks a parity switching of the ground state between a singlet state for $J<J_{c}$ to a doublet state for $J>J_{c}$.[16] For the conventional case, equation (1) shows how $J_{c}$ is independent from the superconducting pairing $\Delta$, depending solely on the density of states at Fermi energy $\pi \frac{S}{2} \rho J_{c}=1$. In comparison, for hydrogenated graphene the parity switching point is $\Delta$ dependent, as can be observed in Fig.3(a). In Fig. 3(b) we plot a contour map of the $\mathrm{BdG}$ spectral function evaluated at $E=0$ as a function of $J$ and $\Delta$, showing a clear linear dependence of $J_{c}$ on $\Delta$. In contrast, in the case of a square lattice, the same procedure yields a $J_{c}$ that is independent of $\Delta$ (inset of Fig.3(b)).

Thus, from an experimental point of view, the parity switching point could be observed by controlling either the superconducting gap $\Delta$, that depends strongly on temperature, as well as tuning the hydrogen magnetic moment by controlling the doping level of graphene with a gate[35]. Given that $E_{S} \simeq \Delta-J / 6$, the critical $J_{c}$ is in the range of the $6 \Delta$, ie, in the range of $10 \mathrm{meV}[42,43]$. Another prediction for experiments is shown in figures $3(\mathrm{c})$ and $3(\mathrm{~d})$, where we show the spectral function of the YSR states, as it would be measured with an STM. This hydrogenated-graphene YSR wave function inherits both the extension and the $C_{3}$ symmetry of the impurity resonance of the normal phase[28] (Fig. 3(c)). In particular, the YSR state peaks on the first neighbors of the hydrogen atom (Fig. 3(d)). 


\subsection{YSR states for superlattices}

We now consider YSR state superlattices formed by several hydrogen atoms chemisorbed in the same sublattice. This secures a ferromagnetic coupling between them[29, 33]. We first consider the case of a dimer (Fig. 4a) which is expected[29] to have $S=1$. The resulting density of states with $\Delta=0$ and $J \neq 0$ shows two peaks (Fig. 4b), rather than only one (Fig. 1d) for the single hydrogen case. When the superconducting pairing is switched on, the YSR spectrum also acquires an extra line, compared to the single hydrogen case. The evolution of the two YSR states is still linear with $J$, as in the single hydrogen case. Thus, there are as many bound YSR states as hydrogen atoms. We now extend this notion to the case of a one dimensional periodic array of hydrogen atoms (Fig. 4d). For this one dimensional YSR crystal we obtain a single YSR band that corresponds to a gapless 1D superconductor, reminiscent of the one recently found at the interface of a magnetically ordered graphene edge and a superconductor[44]. The map of density of states at $E=0$ for the one dimensional array is shown in (Fig.4f), whose spatial profile resembles the single hydrogen YSR state.

So far we assumed that resonant magnetism behaves as a classical magnetic moment. It must be noted that quantum fluctuations scale as $\frac{1}{S}$ and are thus expected to have an important role both in conventional YSR states[45, 46, 47, 48, 49, 50, 51] as well as in the case of hydrogenated graphene[52, 53]. Such fluctuations are not captured within the present theoretical framework. However, our approach becomes more accurate for the larger structures considered in figure 4, that have a larger spin, and thereby smaller quantum fluctuations.
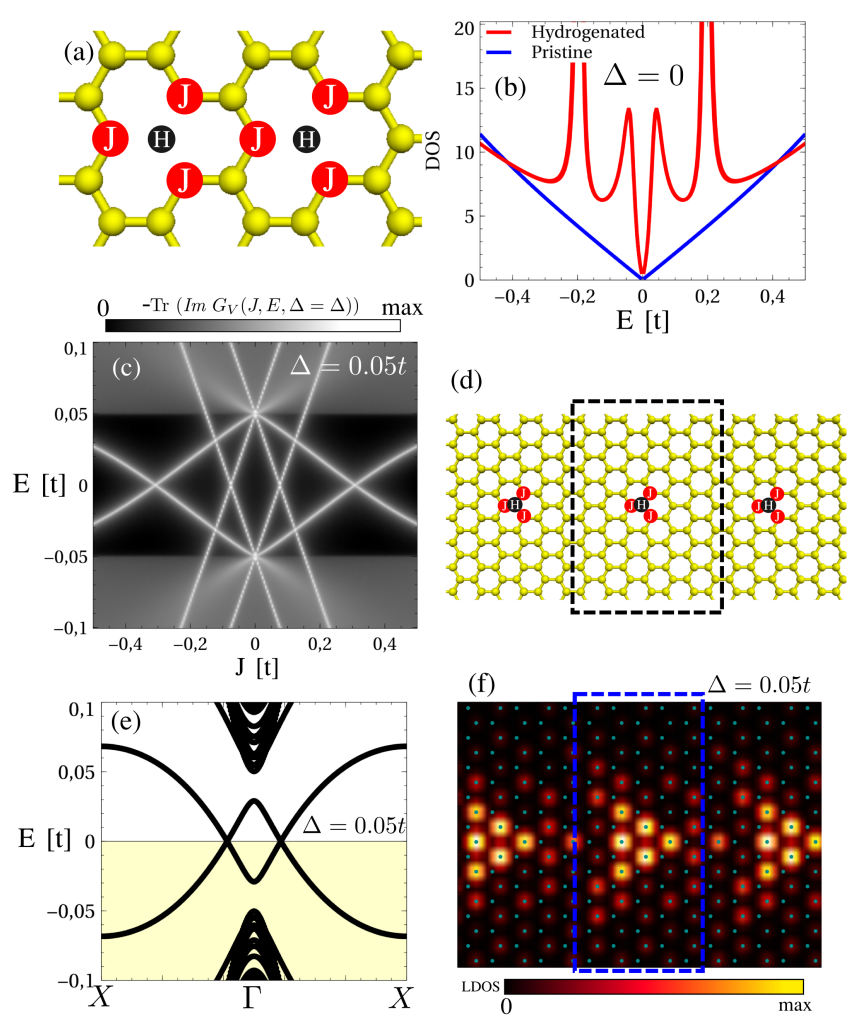

Figure 4. (a) Central cell with two hydrogen atoms, and (b) density of states before the superconducting proximity is considered. (c) Evolution of the YSR spectrum for case (a), resembling the same linear evolution as Fig. 2f. (d) Sketch of the unit cell for a periodic array of hydrogenated sites. Panel (e) shows the BdG band structure of the periodic array, showing a gapless YSR band. Panel (f) shows the spatial resolved DOS for the periodic array (d) at $E=0$.

\section{Conclusions}

We have shown that a single chemisorbed hydrogen in superconducting graphene creates a YSR bound state, in spite of the vanishing density of states of pristine graphene. These YSR states have properties very different from conventional YSR states, such as a linear dependence of the binding energy $E_{S}$ with exchange, and a parity switching point $J_{c}$ that depends on the superconducting pairing energy, and can thereby be modulated with temperature. Motivated by recent experiments that demonstrate the atomic manipulation of 
individual hydrogen atoms on graphene[34], we have also explored the properties of YSR super-structures. Furthermore, these results also apply for a much wider class of covalent functionalizations in graphene[31]. Combined with the electric control of magnetism, this class of systems offers a unique platform to engineer exotic superconducting states at the nanoscale.

\section{Acknowledgments}

JFR acknowledges financial supported by MEC-Spain (FIS2013-47328-C2-2-P) and Generalitat Valenciana (ACOMP/2010/070), Prometeo. This work has been financially supported in part by FEDER funds. We acknowledge financial support by Marie-Curie-ITN 607904SPINOGRAPH. J. L. Lado thanks the hospitality of the Departamento de Fisica Aplicada at the Universidad de Alicante. We thank Ivan Brihuega for sharing experimental data. We thank D. Jacob for bringing to our attention the embedding method[39]. We thank N. Garcia for insightful comments and proof reading of the manuscript. We thank SPICE for support in the organization of the workshop on "Magnetic adatoms as building blocks for quantum magnetism" that has inspired this work.

\section{References}

[1] H Suhl and BT Matthias. Impurity scattering in superconductors. Physical Review, 114(4):977, 1959.

[2] Hiroyuki Shiba. Classical spins in superconductors. Progress of theoretical Physics, 40(3):435451, 1968.

[3] Hiroyuki Shiba and Toshio Soda. Superconducting tunneling through the barrier with paramagnetic impurities. Progress of Theoretical Physics, 41(1):25-44, 1969.
[4] L Yu. Bound state in superconductors with paramagnetic impurities. Acta Phys. Sin, 21:75-91, 1965.

[5] AI Rusinov. Theory of gapless superconductivity in alloys containing paramagnetic impurities. Sov. Phys. JETP, 29(6):1101-1106, 1969.

[6] Ali Yazdani, B. A. Jones, C. P. Lutz, M. F. Crommie, and D. M. Eigler. Probing the local effects of magnetic impurities on superconductivity. 275(5307):1767-1770, 1997.

[7] Shuai-Hua Ji, Tong Zhang, Ying-Shuang Fu, $\mathrm{Xi}$ Chen, Xu-Cun Ma, Jia Li, Wen-Hui Duan, Jin-Feng Jia, and Qi-Kun Xue. Highresolution scanning tunneling spectroscopy of magnetic impurity induced bound states in the superconducting gap of $\mathrm{pb}$ thin films. Physical review letters, 100(22):226801, 2008.

[8] Nino Hatter, Benjamin W Heinrich, Michael Ruby, Jose I Pascual, and Katharina J Franke. Magnetic anisotropy in shiba bound states across a quantum phase transition. Nature communications, 6, 2015.

[9] Gerbold C Ménard, Sébastien Guissart, Christophe Brun, Stéphane Pons, Vasily S Stolyarov, François Debontridder, Matthieu V Leclerc, Etienne Janod, Laurent Cario, Dimitri Roditchev, et al. Coherent long-range magnetic bound states in a superconductor. Nature Physics, 11(12):1013-1016, 2015.

[10] Michael Ruby, Falko Pientka, Yang Peng, Felix von Oppen, Benjamin W Heinrich, and Katharina J Franke. Tunneling processes into localized subgap states in superconductors. Physical review letters, 115(8):087001, 2015.

[11] Andreas Heimes, Panagiotis Kotetes, and Gerd Schön. Majorana fermions from shiba states in an antiferromagnetic chain on top of a superconductor. Physical Review B, 90(6):060507, 2014.

[12] Steven R Elliott and Marcel Franz. Colloquium: Majorana fermions in nuclear, particle, and solid-state physics. Reviews of Modern Physics, 87(1):137, 2015.

[13] Stevan Nadj-Perge, Ilya K Drozdov, Jian Li, Hua Chen, Sangjun Jeon, Jungpil Seo, Allan H MacDonald, B Andrei Bernevig, and Ali Yazdani. Observation of majorana fermions in ferromagnetic atomic chains on a superconductor. Science, 346(6209):602-607, 2014.

[14] Falko Pientka, Leonid I Glazman, and Felix von Oppen. Topological superconducting phase in helical shiba chains. Physical Review B, 
88(15):155420, 2013.

[15] Kim Pöyhönen, Alex Westström, Joel Röntynen, and Teemu Ojanen. Majorana states in helical shiba chains and ladders. Physical Review B, 89(11):115109, 2014.

[16] AV Balatsky, I Vekhter, and Jian-Xin Zhu. Impurity-induced states in conventional and unconventional superconductors. Reviews of Modern Physics, 78(2):373, 2006.

[17] Hubert B Heersche, Pablo Jarillo-Herrero, Jeroen B Oostinga, Lieven MK Vandersypen, and Alberto F Morpurgo. Bipolar supercurrent in graphene. Nature, 446(7131):56-59, 2007.

[18] Katsuyoshi Komatsu, Chuan Li, S AutierLaurent, $\mathrm{H}$ Bouchiat, and $\mathrm{S}$ Guéron. Superconducting proximity effect in long superconductor/graphene/superconductor junctions: From specular andreev reflection at zero field to the quantum hall regime. Physical Review $B, 86(11): 115412,2012$.

[19] Victor E Calado, Srijit Goswami, Gaurav Nanda, Mathias Diez, Anton R Akhmerov, Kenji Watanabe, Takashi Taniguchi, Teun M Klapwijk, and Lieven MK Vandersypen. Ballistic josephson junctions in edge-contacted graphene. arXiv preprint arXiv:1501.06817, 2015.

[20] Charlène Tonnoir, Amina Kimouche, Johann Coraux, Laurence Magaud, Benjamin Delsol, Bruno Gilles, and Claude Chapelier. Induced superconductivity in graphene grown on rhenium. Physical review letters, 111(24):246805, 2013.

[21] Zheng Han, Adrien Allain, Hadi Arjmandi-Tash, Konstantin Tikhonov, Mikhail FeigelMan, Benjamin Sacépé, and Vincent Bouchiat. Collapse of superconductivity in a hybrid tingraphene josephson junction array. Nature Physics, 10(5):380-386, 2014.

[22] Miguel M Ugeda, Aaron J Bradley, Yi Zhang, Seita Onishi, Yi Chen, Wei Ruan, Claudia Ojeda-Aristizabal, Hyejin Ryu, Mark T Edmonds, Hsin-Zon Tsai, et al. Characterization of collective ground states in single-layer nbse 2 . Nature Physics, 12(1):92-97, 2016.

[23] Y. Cao, A. Mishchenko, G. L. Yu, E. Khestanova, A. P. Rooney, E. Prestat, A. V. Kretinin, P. Blake, M. B. Shalom, C. Woods, J. Chapman, G. Balakrishnan, I. V. Grigorieva, K. S. Novoselov, B. A. Piot, M. Potemski, K. Watanabe, T. Taniguchi, S. J. Haigh, A. K. Geim, and R. V. Gorbachev. Quality heterostructures from two-dimensional crystals unstable in air by their assembly in inert atmosphere. Nano Letters, 15(8):4914-4921, 2015.

[24] DK Efetov, L Wang, C Handschin, KB Efetov, J Shuang, R Cava, T Taniguchi, K Watanabe, J Hone, CR Dean, et al. Specular interband andreev reflections in graphene. arXiv preprint arXiv:1505.04812, 2015.

[25] S-L Yang, JA Sobota, CA Howard, CJ Pickard, Makoto Hashimoto, DH Lu, S-K Mo, PS Kirchmann, and Z-X Shen. Superconducting graphene sheets in cac6 enabled by phononmediated interband interactions. Nature communications, 5, 2014.

[26] J Chapman, Y Su, CA Howard, D Kundys, A Grigorenko, F Guinea, AK Geim, IV Grigorieva, and RR Nair. Superconductivity in cadoped graphene. Nature Physics, 5, 2014.

[27] KC Rahnejat, CA Howard, NE Shuttleworth, SR Schofield, K Iwaya, CF Hirjibehedin, Ch Renner, G Aeppli, and M Ellerby. Charge density waves in the graphene sheets of the superconductor cac $_{6}$. Nature communications, 2:558, 2011.

[28] Oleg V Yazyev and Lothar Helm. Defect-induced magnetism in graphene. Physical Review B, 75(12):125408, 2007.

[29] J. J. Palacios, J. Fernández-Rossier, and L. Brey. Vacancy-induced magnetism in graphene and graphene ribbons. Phys. Rev. B, 77:195428, May 2008.

[30] RR Nair, M Sepioni, I-Ling Tsai, O Lehtinen, J Keinonen, AV Krasheninnikov, T Thomson, AK Geim, and IV Grigorieva. Spin-half paramagnetism in graphene induced by point defects. Nature Physics, 8(3):199-202, 2012.

[31] Elton JG Santos, Andrés Ayuela, and Daniel Sánchez-Portal. Universal magnetic properties of sp3-type defects in covalently functionalized graphene. New Journal of Physics, 14(4):043022, 2012.

[32] DW Boukhvalov, MI Katsnelson, and AI Lichtenstein. Hydrogen on graphene: Electronic structure, total energy, structural distortions and magnetism from first-principles calculations. Physical Review B, 77(3):035427, 2008.

[33] D Soriano, Federico Muñoz-Rojas, J. FernándezRossier, and JJ Palacios. Hydrogenated graphene nanoribbons for spintronics. Physical Review B, 81(16):165409, 2010.

[34] Ivan Brihuega. Atomic scale control of graphene 
magnetism using hydrogen atoms. SPICE workshop, Magnetic adatoms as building blocks for quantum magnetism, 2015.

[35] RR Nair, I-L Tsai, M Sepioni, O Lehtinen, J Keinonen, AV Krasheninnikov, AH Castro Neto, MI Katsnelson, AK Geim, and IV Grigorieva. Dual origin of defect magnetism in graphene and its reversible switching by molecular doping. Nature communications, 4, 2013.

[36] Felix Yndurain. Effect of hole doping on the magnetism of point defects in graphene: A theoretical study. Physical Review B, 90(24):245420, 2014.

[37] TO Wehling, AV Balatsky, MI Katsnelson, AI Lichtenstein, K Scharnberg, and R Wiesendanger. Local electronic signatures of impurity states in graphene. Physical Review B, 75(12):125425, 2007.

[38] CWJ Beenakker. Specular andreev reflection in graphene. Physical review letters, 97(6):067007, 2006.

[39] D Jacob and G Kotliar. Orbital selective and tunable kondo effect of magnetic adatoms on graphene: Correlated electronic structure calculations. Physical Review B, 82(8):085423, 2010.

[40] NMR Peres, F Guinea, and AH Castro Neto. Electronic properties of disordered two-dimensional carbon. Physical Review B, 73(12):125411, 2006.

[41] TO Wehling, HP Dahal, AI Lichtenstein, and AV Balatsky. Local impurity effects in superconducting graphene. Physical Review B, 78(3):035414, 2008.

[42] WL McMillan. Tunneling model of the superconducting proximity effect. Physical Review, 175(2):537, 1968.

[43] A Chrestin, $\mathrm{T}$ Matsuyama, and $\mathrm{U}$ Merkt. Evidence for a proximity-induced energy gap in nb/inas/nb junctions. Physical Review B, 55(13):8457, 1997.

[44] P. San-Jose, J. L. Lado, R. Aguado, F. Guinea, and J. Fernández-Rossier. Majorana zero modes in graphene. Phys. Rev. X, 5:041042, Dec 2015.

[45] Jong Soo Lim, Rosa López, Ramón Aguado, et al. Shiba states and zero-bias anomalies in the hybrid normal-superconductor anderson model. Physical Review B, 91(4):045441, 2015.

[46] Tamifusa Matsuura. The effects of impurities on superconductors with kondo effect. Progress of
Theoretical Physics, 57(6):1823-1835, 1977.

[47] Yosuke Nagaoka and Tamifusa Matsuura. Bound states due to a magnetic impurity in a superconductor. i. Progress of Theoretical Physics, 46(2):364-386, 1971.

[48] Toshio Soda, Tamifusa Matsuura, and Yosuke Nagaoka. sd exchange interaction in a superconductor. Progress of Theoretical Physics, 38(3):551-567, 1967.

[49] Koji Satori, Hiroyuki Shiba, Osamu Sakai, and Yukihiro Shimizu. Numerical renormalization group study of magnetic impurities in superconductors. Journal of the Physical Society of Japan, 61(9):3239-3254, 1992.

[50] Johannes Bauer, A Oguri, and AC Hewson. Spectral properties of locally correlated electrons in a bardeen-cooper-schrieffer superconductor. Journal of Physics: Condensed Matter, 19(48):486211, 2007.

[51] Gediminas Kiršanskas, Moshe Goldstein, Karsten Flensberg, Leonid I Glazman, and Jens Paaske. Yu-shiba-rusinov states in phase-biased superconductor-quantum dot-superconductor junctions. Physical Review B, 92(23):235422, 2015.

[52] P Haase, S Fuchs, T Pruschke, H Ochoa, and $\mathrm{F}$ Guinea. Magnetic moments and kondo effect near vacancies and resonant scatterers in graphene. Physical Review B, 83(24):241408, 2011.

[53] JO Sofo, Gonzalo Usaj, PS Cornaglia, AM Suarez, AD Hernández-Nieves, and CA Balseiro. Magnetic structure of hydrogen-induced defects on graphene. Physical Review B, 85(11):115405, 2012. 